\title{
2 Photogranulation in a Hydrostatic Environment Occurs with Limitation of
}

\section{Iron}

4 Abeera A. Ansari ${ }^{\dagger}+$, Arfa A. Ansari ${ }^{\dagger}$, Ahmed S. Abouhend ${ }^{\dagger}$, Joseph G. Gikonyo ${ }^{\dagger}$ and Chul 5 Park $^{\dagger^{*}}$

6

$7 \dagger$ Department of Civil and Environmental Engineering, University of Massachusetts, Amherst, 8 MA 01002, USA

$9 \quad$ U.S.-Pakistan Center for Advanced Studies in Energy (USPCAS-E), National University of 10 Sciences \& Technology (NUST), H-12 Sector (44000), Islamabad, Pakistan

\section{Summary}

16 Number of pages: 13 (S1-S13)

17 Number of Tables: 2

18 Number of Figures: 7 
Detailed Materials and Methods

27 Bulk liquid filtration for Fe. Fe in bulk liquid passing through Ultracel 30 kDa ultra-filtration membrane (EMD Millipore Corporation, Billerica, MA, USA) was considered as dissolved Fe. ${ }^{32}$ Bulk liquid was also passed through $0.45 \mu \mathrm{m}$ mixed cellulose ester membrane (Fisher Scientific, USA). Fe in the size fraction smaller than $0.45 \mu \mathrm{m}$ and greater than $30 \mathrm{kDa}$ was considered colloidal Fe.

\section{Estimation of phycobilin concentration in photogranular biomass. Ten $\mathrm{mL}$ mixed biomass} sample was centrifuged at $12000 \mathrm{rpm}$ for $10 \mathrm{~min}$. After discarding the supernatant, the pellet was re-suspended in $0.025 \mathrm{M}$ phosphate buffer saline solution ( $\mathrm{pH}$ 7.2). The sample was then subjected to homogenization at $700 \mathrm{rpm}$ for $1 \mathrm{~min}$ (IKA T18 basic Ultra-Turrax), followed by sonication (Fisher Scientific Sonic Dismembrator Model 500) at 20\% strength for 2 min. After centrifugation, the obtained supernatant was filtered through $0.22 \mu \mathrm{m}$ syringe filter (Basix, Fisher Scientific) and its absorbance was read at $566 \mathrm{~nm}, 620 \mathrm{~nm}, 652 \mathrm{~nm}$, and $750 \mathrm{~nm}$ using a portable spectrometer (DR 2700, Hach, US). Absorbance $\lambda_{\max }$ for phycoerythrin, phycocyanin and allophycocyanin was obtained at $566 \mathrm{~nm}, 620 \mathrm{~nm}$, and $652 \mathrm{~nm}$, respectively, by using phycobilin standards (Prozyme, US). Absorbance at $750 \mathrm{~nm}$ represented the background interference, which was subtracted from other wavelengths. ${ }^{39}$ Corrected $566 \mathrm{~nm}$, $620 \mathrm{~nm}$, and $652 \mathrm{~nm}$ absorbances were introduced in the Bennett and Bogorad equations ${ }^{37}$ to quantify the concentrations of respective phycobilin.

\section{Extraction of extracellular polymeric substances (EPS) from photogranular biomass. A} twenty $\mathrm{mL}$ biomass sample was centrifuged at $12000 \mathrm{rpm}$ for $10 \mathrm{~min}$ at $4{ }^{\circ} \mathrm{C}$. The resulting

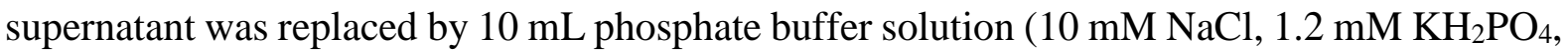
and $6 \mathrm{mM} \mathrm{Na}_{2} \mathrm{HPO}_{4}$ ), after which the sample was proceeded to homogenization at $700 \mathrm{rpm}$ for 
$5130 \mathrm{~s}$ (IKA T18 basic Ultra-Turrax) and sonication (Fisher Scientific Sonic Dismembrator

52 Model 500) at 10\% strength for $40 \mathrm{~s}$. The sample was centrifuged, and the resulting supernatant

53 was filtered through $0.45 \mu \mathrm{m}$ cellulose filter to obtain EPS extract by sonication. The remaining

54 pellet was subjected to base treatment by re-suspending it in $10 \mathrm{~mL}$ phosphate buffer solution

55 and bringing its $\mathrm{pH}$ to $10.5-11$ using $1 \mathrm{M} \mathrm{NaOH} .{ }^{33}$ The sample was then shaken at $425 \mathrm{rpm}$ for

$562 \mathrm{~h}$ at $4{ }^{\circ} \mathrm{C}$ and centrifuged. The supernatant was filtered through $0.45 \mu \mathrm{m}$ cellulose filter to get

57 the EPS extract by base treatment. ${ }^{33}$

58

59

60

61

62

63

64

65

66

67

68

69

70

71

72

73

74

75 
76 Table S1. Pearson correlation between phycobilin and other photosynthetic pigments

77 produced during cultivations.

\begin{tabular}{cccc}
\hline & \multicolumn{3}{c}{ Phycobilin } \\
\hline & Amherst & Hadley & Springfield \\
\hline Chlorophyll a & 0.94 & 0.79 & 0.70 \\
Chlorophyll b & 0.64 & 0.63 & 0.70 \\
Chlorophyll c & 0.51 & 0.27 & 0.66 \\
\hline
\end{tabular}

79 
80 Table S2. Pearson correlation between Fe and organic matter in bulk liquid.

\begin{tabular}{|c|c|c|c|c|c|c|c|}
\hline & & \multicolumn{2}{|c|}{ Amherst } & \multicolumn{2}{|l|}{ Hadley } & \multicolumn{2}{|c|}{ Springfield } \\
\hline & & Colloidal Fe & Dissolved Fe & Colloidal Fe & $\begin{array}{c}\text { Dissolved } \\
\mathrm{Fe}\end{array}$ & Colloidal Fe & Dissolved Fe \\
\hline & & $30 \mathrm{kDa}<\mathrm{Fe}<0.45 \mu \mathrm{m}$ & $\mathrm{Fe}<30 \mathrm{Kda}$ & $30 \mathrm{kDa}<\mathrm{Fe}<0.45 \mu \mathrm{m}$ & $\mathrm{Fe}<30 \mathrm{Kda}$ & $30 \mathrm{kDa}<\mathrm{Fe}<0.45 \mu \mathrm{mm}$ & $\mathrm{Fe}<30 \mathrm{Kda}$ \\
\hline & Colloidal bulk liquid fraction & -0.86 & -0.62 & -0.31 & -0.01 & 0.06 & 0.14 \\
\hline $\begin{array}{l}\text { Dissolved organic nitrogen } \\
\text { (DON) }\end{array}$ & Dissolved bulk liquid fraction & 0.29 & 0.06 & 0.07 & -0.32 & -0.18 & -0.50 \\
\hline & Colloidal bulk liquid fraction & -0.58 & -0.23 & -0.04 & 0.13 & 0.10 & 0.13 \\
\hline $\begin{array}{l}\text { Dissolved organic carbon } \\
\text { (DOC) }\end{array}$ & Dissolved bulk liquid fraction & -0.46 & -0.51 & -0.29 & -0.55 & -0.54 & -0.76 \\
\hline
\end{tabular}




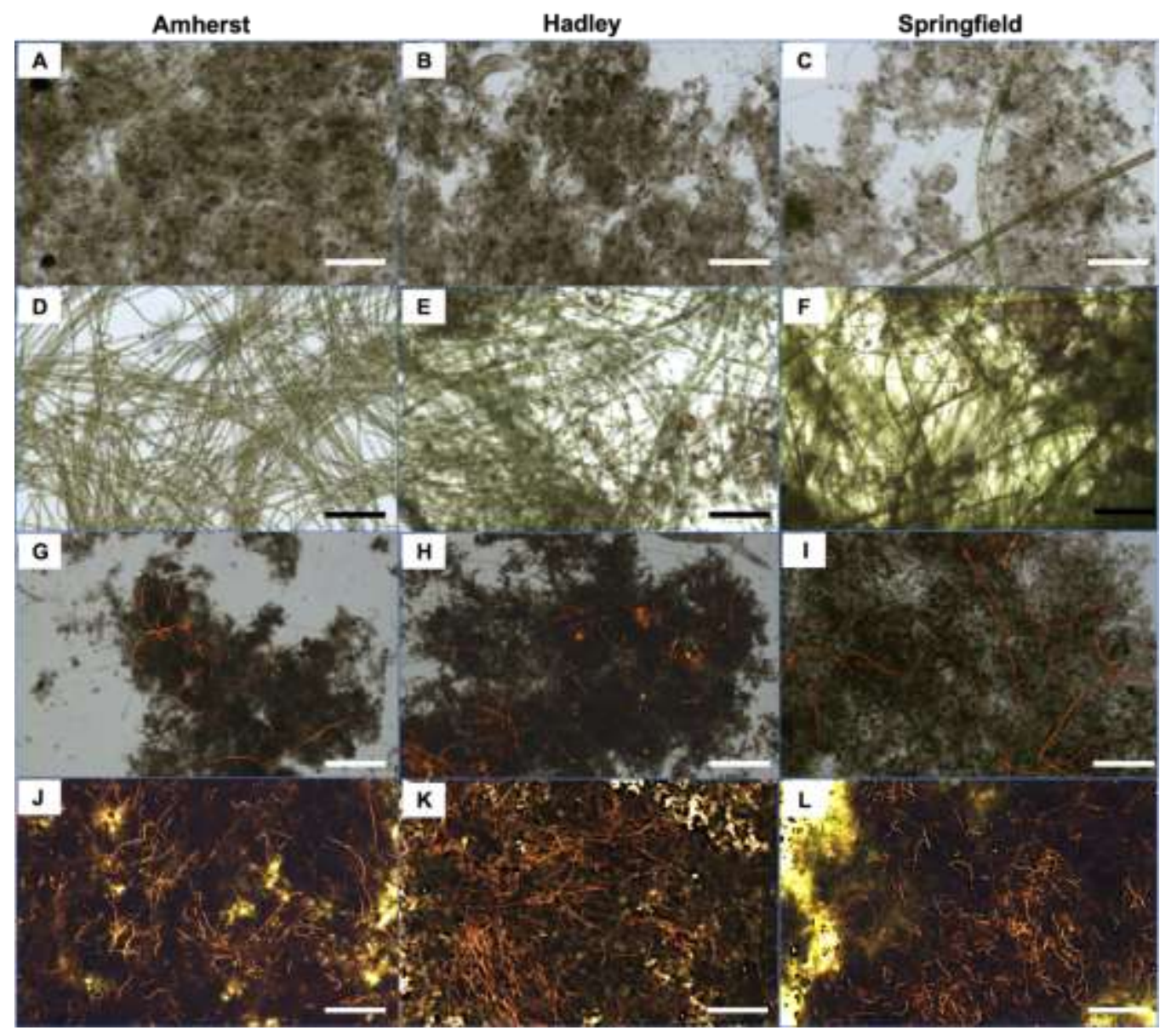

84 Figure S1. Microscopy of samples during hydrostatic cultivation of photogranules. Panels Aalgae in the top layer of settled sludge biomass. Panels D-F (between day 9-12, brightfield microscopy) display outgrowth of filamentous green algae. Panels G-I (between day 9-12, autofluorescence microscopy) illustrate the appearance of cyanobacteria (indicated by goldenorange fluorescence) in top layer of settled biomass. Panels J-L (between day 15-18, autofluorescence microscopy) shows the growth of filamentous cyanobacteria which eventually dominated the phototrophic community. Scale bars for panels A-F and I are 400 $\mu \mathrm{m}$; scale bars for panels G-H and J-L are $2000 \mu \mathrm{m}$. 

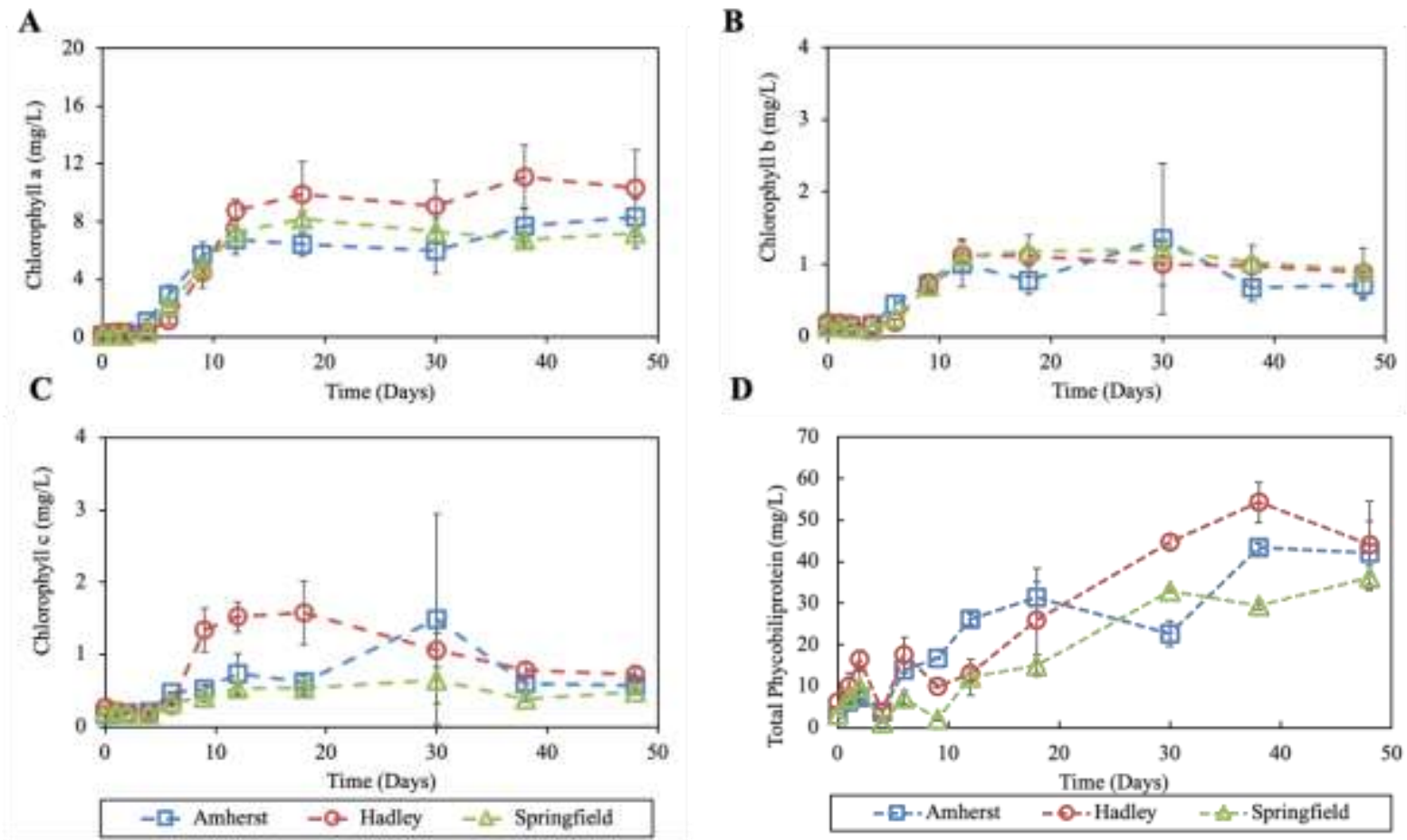

97 Figure S2. Changes in photopigment concentrations during the progression of

98 photogranulation under hydrostatic conditions using three different activated sludges as inoculum. (A) chlorophyl a, (B) chlorophyll b, (C) chlorophyll c, and (D) total phycobiliprotein. Error bars represent standard deviation from triplicate samples. 

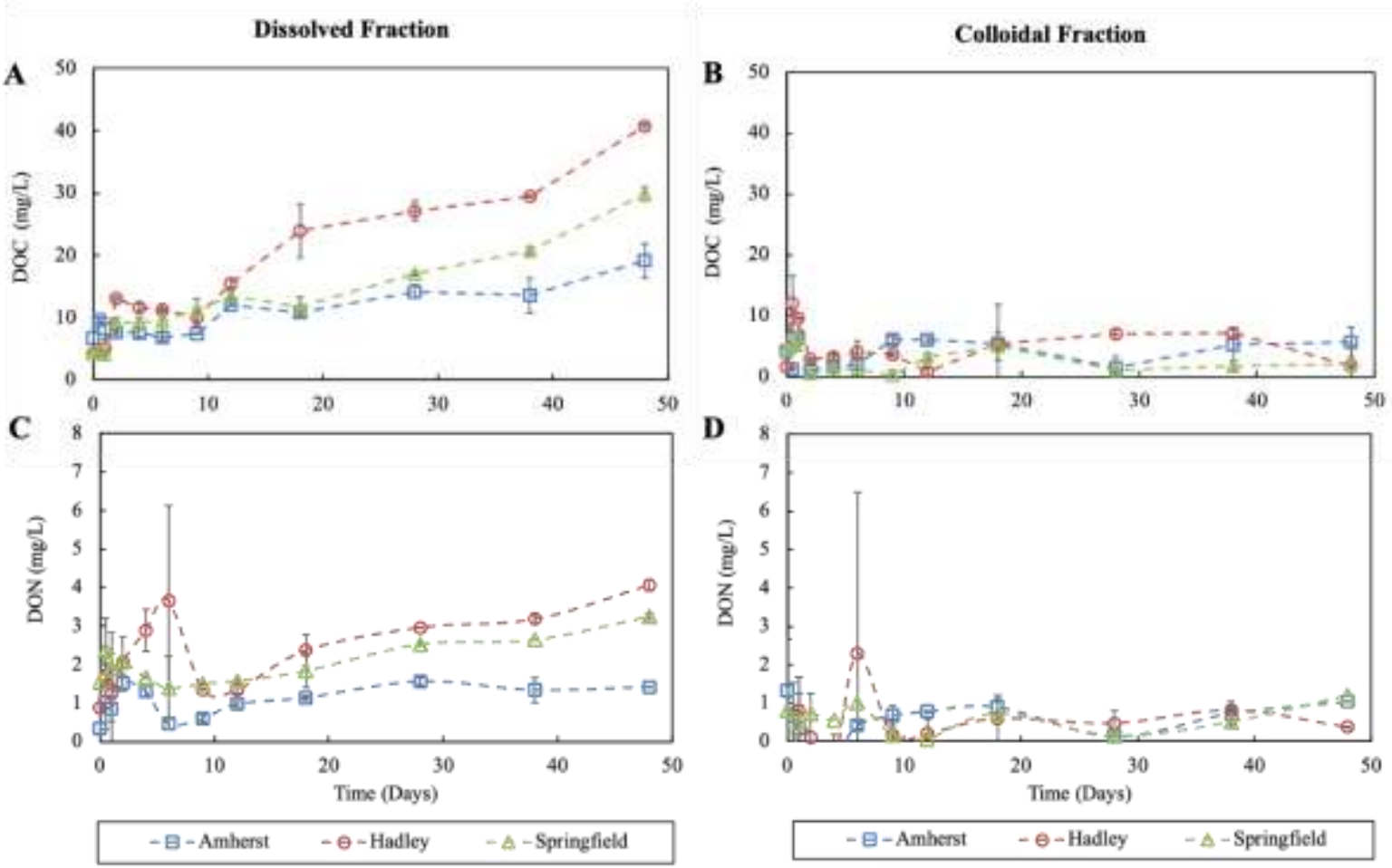

102

Figure S3. Changes in dissolved organic carbon (DOC) (A, B) and dissolved organic nitrogen

104 (DON) (C, D) in the fractions of dissolved bulk liquid (<30 kDa) and colloidal bulk liquid

$105(0.45 \mu \mathrm{m}-30 \mathrm{kDa})$ during the progression of photogranulation under hydrostatic conditions.

106 Error bars represent the range of results from duplicate samples. 


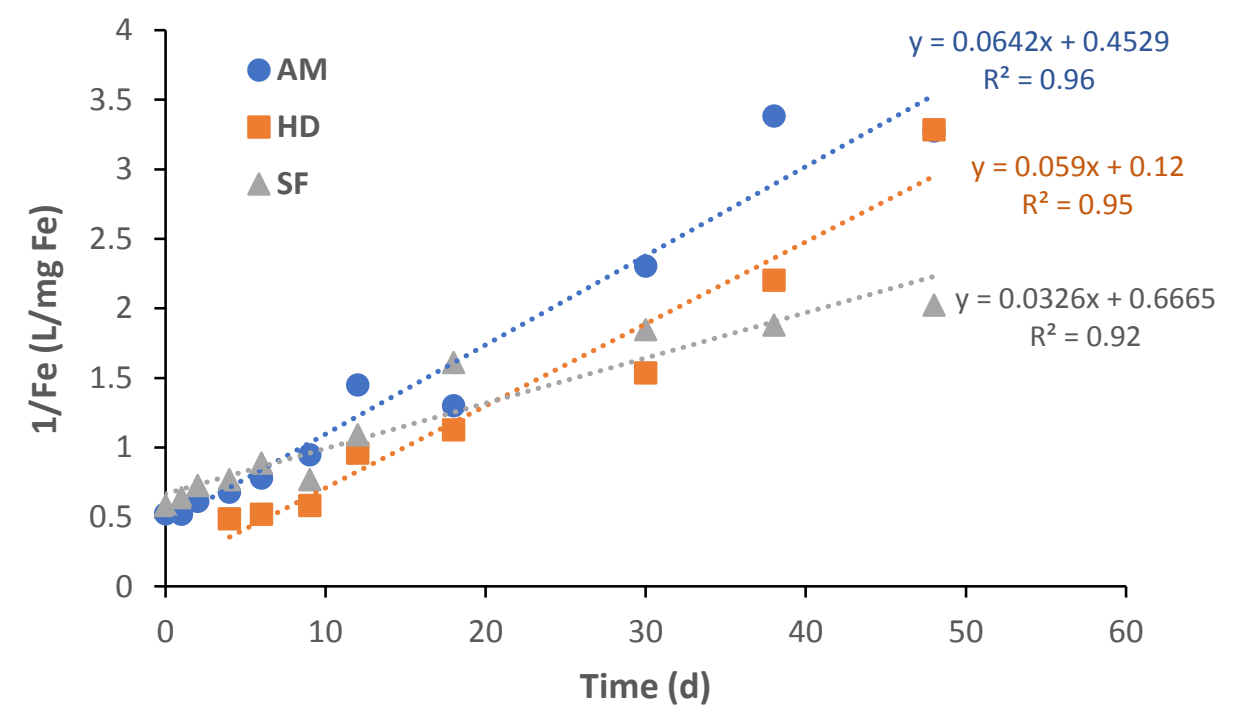

108

109 Figure S4. Curve fitting analysis showing second-order reaction of Fe $\mathrm{EPS}_{\text {in }}$ in hydrostatic 110 cultivation.

111 

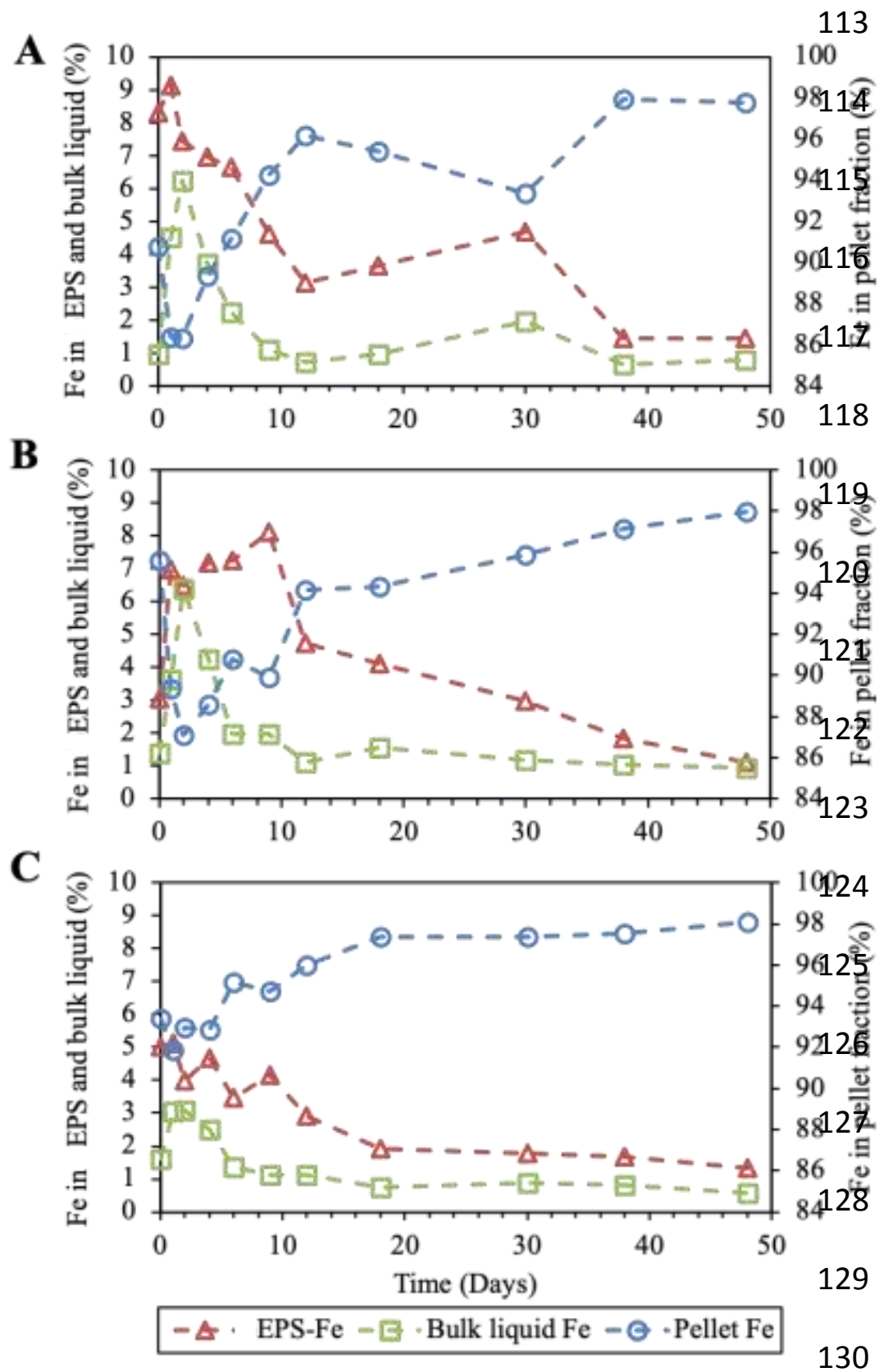

132 Figure S5. Distribution of $\mathrm{Fe}(\%)$ in different fractions of biomass during the photogranulation

133 process in a hydrostatic environment. Activated sludge inoculums: (A) Amherst, (B) Hadley, 134 and (C) Springfield activated sludges. 


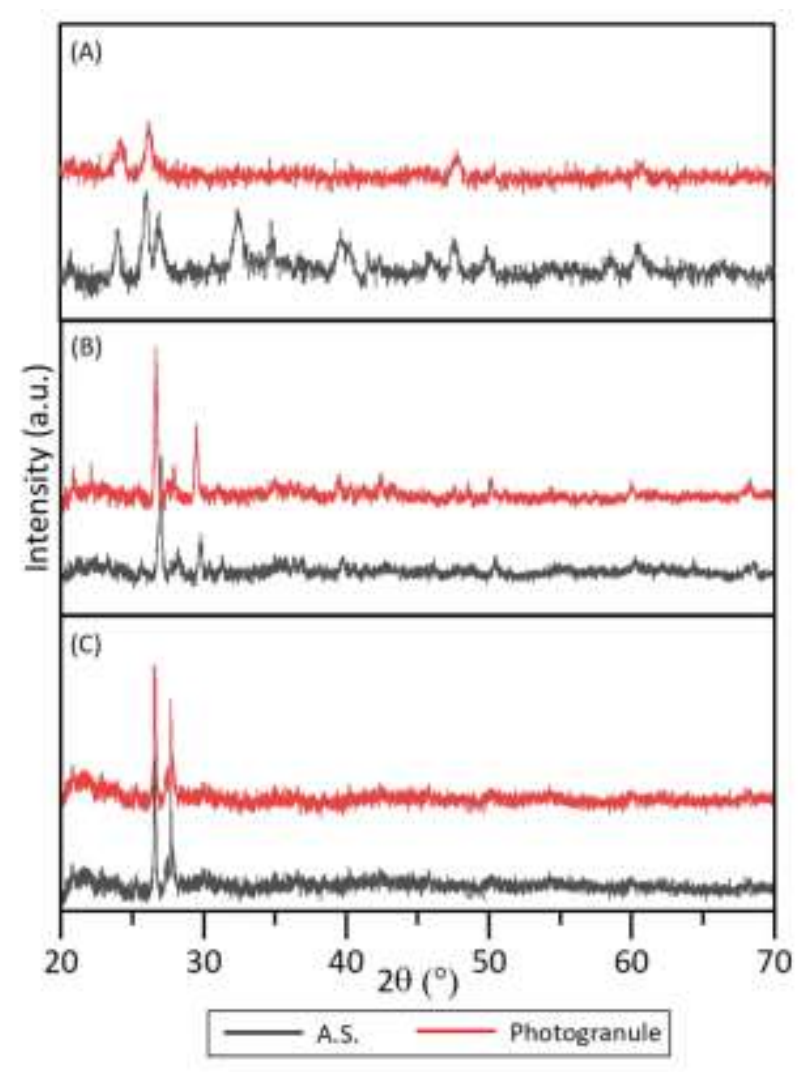

138 Figure S6. Powder X-Ray diffraction patterns of activated sludge (A.S.) and OPG 139 (photogranule) in hydrostatic cultivations of (A) Amherst, (B) Hadley, and (C) Springfield sets.

140 The results show the existence of iron mostly in amorphous forms. We did not observe a

141 complete diffraction pattern for any known crystalline ferric oxide. Furthermore, many oxides

142 have overlapping diffraction patterns which made PXRD analysis complicated. 
Figure S7 depicts the FTIR spectra of activated sludge and OPG biomass. The typical

$\mathrm{O}-\mathrm{H}$ broad stretching peak centered at $3300 \mathrm{~cm}^{-1}$ (Peak 1) suggests the formation of ferric oxyhydroxide $\mathrm{Fe}(\mathrm{O}) \mathrm{OH}$ and/or ferric hydroxides $\mathrm{Fe}(\mathrm{OH})_{3}$ in both activated sludge and $\mathrm{OPG}$ samples. The progression of OPG occurred, after initial anaerobic conditions, in oxic (Figure 4) and circumneutral $\mathrm{pH}(6.5-8)$ environment, which is known to favor the formation of insoluble ferric hydroxides. Swanner et al. ${ }^{53}$ stated that cyanobacterium Synechococcus PCC 7002 oxidized Fe (II) to ferric oxyhydroxide via chemical oxidation using photosynthetic oxygenation. The O-H group stretches can also potentially belong to the carboxylic group of polysaccharides (PS). These FTIR results along with earlier correlation analysis between bEPS-PS and Fe might indicate possible complexation between Fe (III) and PS. The interactions between proteins (PN) and Fe (III) were also depicted in FTIR spectra. Peaks related to PN were found at $1637-1640 \mathrm{~cm}^{-1}$ (Peak 2), 1531-1541 $\mathrm{cm}^{-1}$ (Peak 3) and 1225$1233 \mathrm{~cm}^{-1}$ (Peak 4), which corresponded to amide $\mathrm{C}=\mathrm{O}$ carbonyl group, amide $\mathrm{II}(\mathrm{N}-\mathrm{H})$ double bond, and $\mathrm{C}-\mathrm{O}$ stretching bond of $\mathrm{COOH}$, respectively. ${ }^{56,57}$ These peaks decreased in intensity during the photogranulation process. The decline in these functional groups seems to support the decrease in EPS-PN, which was also strongly correlated with Fe $\mathrm{EPS}_{\text {in }}$ in this study. Apart from EPS-PN, FTIR spectra also displayed the relative increase in C-O stretching bond of PS (1024-1066 $\mathrm{cm}^{-1}$, Peak 5) in Hadley. Earlier studies showed that cyanobacteria accumulate Fe 173 precipitates on their outer polysaccharide sheath. ${ }^{54,55}$ The increasing EPS-PS content could, 174 therefore, possibly contain Fe either as insoluble Fe (III) precipitate (accumulation via 175 adsorption) or Fe complexation with carbonyl/carboxylic groups.

176 

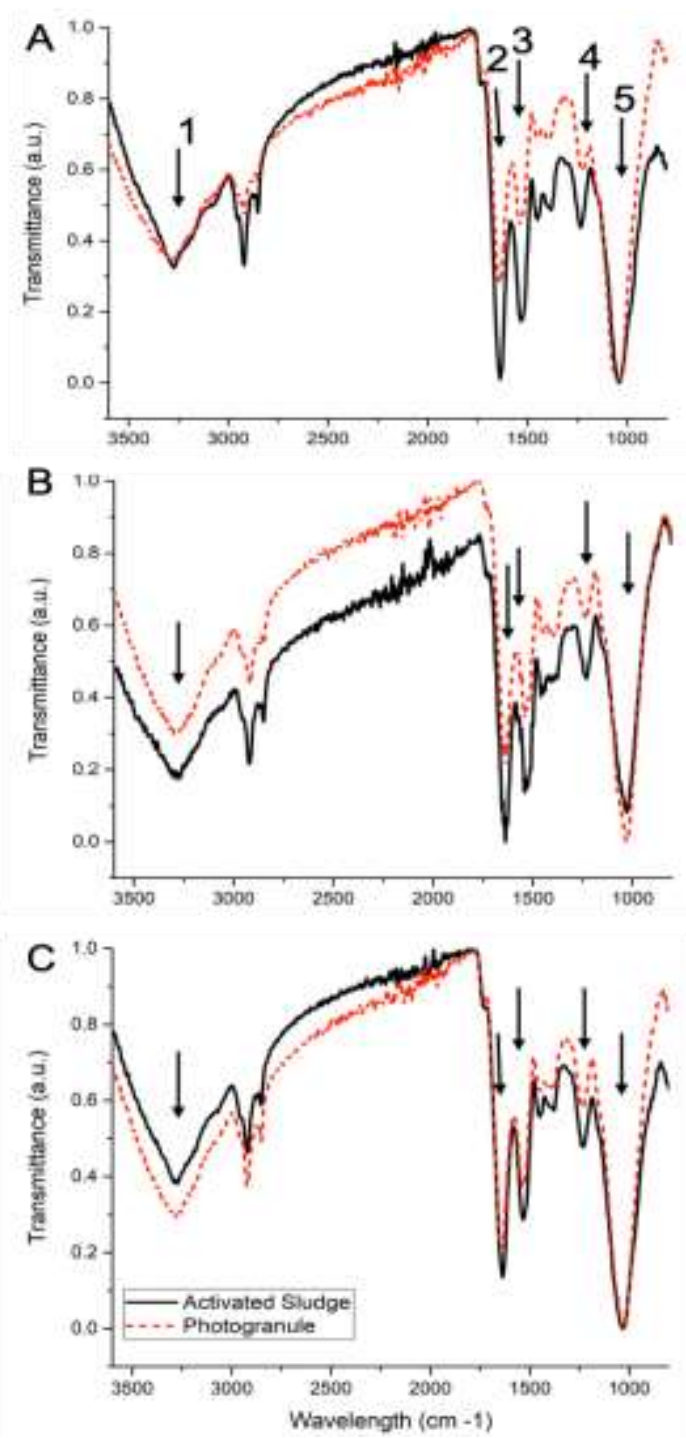

179 Figure S7. Room-temperature FTIR spectra of activated sludge (A.S.) and OPG 180 (photogranule) in hydrostatic cultivations of (A) Amherst, (B) Hadley, and (C) Springfield sets. 181 Peak 1 (centered at $3300 \mathrm{~cm}^{-1}$ ) shows O-H bond stretching, Peak $2\left(1637-1640 \mathrm{~cm}^{-1}\right)$ shows 182 amide $\mathrm{C}=\mathrm{H}$ carbonyl group band, Peak $3\left(1531-1541 \mathrm{~cm}^{-1}\right)$ shows $\mathrm{N}-\mathrm{H}$ (2nd-amide) double 183 bond, Peak $4\left(1225-1233 \mathrm{~cm}^{-1}\right)$ shows C-O stretching band or COOH, and Peak 5 (1024-1066 $184 \mathrm{~cm}^{-1}$ ) belongs to C-O stretching bond. 